\title{
Gamma-ray spectroscopy for probing highly radioactive items behind thick shields?
}

\author{
D J S Findlay, G P Škoro and G J Burns
}

\section{Published version information}

Citation: DJS Findlay, G Skoro and GJ Burns. "Gamma-ray spectroscopy for probing highly radioactive items behind thick shields?" Journal of Radiological Protection, vol. 38 (2018): N36.

DOI: $\underline{10.1088 / 1361-6498 / a a d 2 e 6}$

This is an author-created, un-copyedited version of an article accepted for publication in Journal of Radiological Protection. The publisher is not responsible for any errors or omissions in this version of the manuscript or any version derived from it. The Version of Record is available online at DOI above. This article is licensed under https://creativecommons.org/licenses/by-nc-nd/3.0/

This version is made available in accordance with publisher policies. Please cite only the published version using the reference above. This is the citation assigned by the publisher at the time of issuing the AAM. Please check the publisher's website for any updates. 


\title{
Gamma-ray spectroscopy for probing highly radioactive items behind thick shields?
}

\author{
D J S Findlay, G P Škoro and G J Burns \\ ISIS, STFC, Rutherford Appleton Laboratory, Oxfordshire OX11 0QX, UK
}

\begin{abstract}
In favourable circumstances, including the availability of prior knowledge, a potential use of gamma-ray spectroscopy with an HPGe detector for probing highly radioactive items behind thick shields is described.
\end{abstract}

\section{Introduction}

For decades there has been much interest in measuring quantities of radioactive material remotely [1]. Techniques for making such measurements tend to fall into two broad categories: 'active' techniques using machine-generated particles to evoke a measurable response from the material, and 'passive' techniques whereby particles spontaneously emitted from the material are measured directly. Since passive measurements naturally become more difficult to make as shielding around the material becomes thicker, measurements through thick shields are usually made using active techniques driven by gamma-rays and/or neutrons from particle accelerators. Nevertheless, it has been shown that for radionuclides emitting several high-energy gamma-rays it is possible to use the relative strengths of gamma-ray lines measured passively outside a thick shield to make good estimates of the thickness of the shield and therefore to determine the absorption corrections necessary to quantify activities behind the shield [2].

In the present publication we apply the method to the particular case of highly radioactive material inside a transportation flask with thick lead walls, and we show that in favourable circumstances and with the availability of prior knowledge account can successfully be taken of simple representations of heterogeneity in the spatial distribution of activity behind the shield.

\section{Example and discussion}

When three proton targets from Target Station 2 (TS-2) on the ISIS Spallation Neutron Source [3] were being prepared for transportation off site for interim storage and ultimate disposal, several gamma-ray spectra were recorded with a Canberra Falcon 5000 HPGe detector [4] $50 \mathrm{~cm}$ from the surface of the cylindrical flask, the longest being an over-weekend spectrum with real and live times of 233946 and 233353 seconds respectively. The spectrum is shown in Figure 1, and count rates of the gamma-ray lines observed in the spectrum are given in Table 1. Because the attenuation of gamma-rays passing through a thick lead shield depends very strongly on energy, i.e. because $\exp (-\mu / \rho(k) \rho x)$ varies rapidly ${ }^{1}$ with gamma-ray energy $k$

\footnotetext{
${ }^{1}$ At $800,1000,1250$ and $1500 \mathrm{keV}$ mass attenuation coefficients for gamma-rays in lead are 0.0887 , $0.0710,0.0588$ and $0.0522 \mathrm{~cm}^{2} \mathrm{~g}^{-1}$ respectively [5]. For $21 \mathrm{~cm}$ of lead with density $11.3 \mathrm{~g} \mathrm{~cm}^{-3}$, the
} 
when $x$ is large (where $\mu / \rho=\mu / \rho(k)$ ) is the mass attenuation coefficient $\left(\mathrm{cm}^{2} \mathrm{~g}^{-1}\right.$ ) for gamma-rays in lead, $\rho$ is the density $\left(\mathrm{g} \mathrm{cm}^{-3}\right)$ of lead, and $x$ is the thickness $(\mathrm{cm})$ of lead), the $1093.63-\mathrm{keV}$ line from ${ }^{172} \mathrm{Lu}$ is lowest-energy gamma-ray line that could reliably be seen above background in the measured spectrum. The strong dependence of attenuation on energy is also the reason why the count rates for the higher-energy ${ }^{172} \mathrm{Lu}$ lines are noticeably greater than might be expected from the gamma-ray emission probabilities (or 'abundances') of the gamma-ray lines.

\begin{tabular}{|ccccc|}
\hline Ident. & $\mathrm{keV}$ & $\%$ & $\mathrm{cps}$ & $\pm \mathrm{cps}$ \\
$\mathrm{Pb} \mathrm{K} \alpha 2$ & 72.80 & & & \\
$\mathrm{~Pb} \mathrm{~K} \alpha 1$ & 74.97 & & & \\
$\mathrm{Th}$ & 238.63 & & & \\
$\mathrm{Ra} / \mathrm{Rn}$ & 295.22 & & & \\
$\mathrm{Ra} / \mathrm{Rn}$ & 351.93 & & & \\
511 & 511.00 & & & \\
$\mathrm{Th}$ & 583.19 & & & \\
$\mathrm{Ra} / \mathrm{Rn}$ & 609.32 & & & \\
$\mathrm{Ra} / \mathrm{Rn}$ & 768.36 & & & \\
$\mathrm{Th}$ & 911.20 & & & \\
$\mathrm{Th}$ & 968.96 & & & \\
$\mathrm{Lu}-172$ & 1093.63 & 62.50 & 0.3348 & 0.0037 \\
$\mathrm{Lu}-172$ & 1387.18 & 0.93 & 0.0403 & 0.0019 \\
$\mathrm{Lu}-172$ & 1397.50 & 0.28 & 0.0094 & 0.0008 \\
$\mathrm{Lu}-172$ & 1402.53 & 0.72 & 0.0402 & 0.0025 \\
$\mathrm{Lu}-172$ & 1440.38 & 0.60 & 0.0347 & 0.0019 \\
$\mathrm{~K}-40$ & 1460.82 & & & \\
$\mathrm{Lu}-172$ & 1465.98 & 0.67 & 0.0455 & 0.0019 \\
$\mathrm{Lu}-172$ & 1470.39 & 0.71 & 0.0512 & 0.0019 \\
$\mathrm{Lu}-172$ & 1488.94 & 1.15 & 0.0835 & 0.0018 \\
$\mathrm{Lu}-172$ & 1542.85 & 1.02 & 0.0956 & 0.0017 \\
$\mathrm{Lu}-172$ & 1584.12 & 2.64 & 0.2772 & 0.0020 \\
$\mathrm{Lu}-172$ & 1602.54 & 0.30 & 0.0325 & 0.0015 \\
$\mathrm{Lu}-172$ & 1608.81 & 0.11 & 0.0135 & 0.0019 \\
$\mathrm{Lu}-172$ & 1621.92 & 2.16 & 0.2461 & 0.0014 \\
$\mathrm{Lu}-172$ & 1666.84 & 0.28 & 0.0286 & 0.0006 \\
$\mathrm{Lu}-172$ & 1670.49 & 0.53 & 0.0703 & 0.0018 \\
$\mathrm{Lu}-172$ & 1724.35 & 0.44 & 0.0708 & 0.0013 \\
$\mathrm{Ra} / \mathrm{Rn}$ & 1764.49 & & & \\
$\mathrm{Lu}-172$ & 1812.85 & 0.19 & 0.0352 & 0.0011 \\
$\mathrm{Lu}-172$ & 1914.80 & 0.60 & 0.1408 & 0.0013 \\
$\mathrm{Lu}-172$ & 1994.36 & 0.15 & 0.0323 & 0.0006 \\
$\mathrm{Lu}-172$ & 2024.90 & 0.06 & 0.0152 & 0.0003 \\
\hline & & & & \\
\hline
\end{tabular}

Table 1. Measured line strengths (counts per second) in over-weekend gamma-ray spectrum described in the text from lead transportation flask containing three spent TS-2 targets. '\%' denotes the emission probability (or 'abundance') of each gamma-ray line.

attenuation factors at 800, 1000, 1250 and $1500 \mathrm{keV}$ are 7.22E-10, 4.80E-08, 8.80E-07 and 4.15E-06 respectively — variation by a factor $\sim 6000$ over the range $800-1500 \mathrm{keV}$. 
The three targets within the transportation flask are essentially solid tungsten cylinders (axes vertical) $30 \mathrm{~cm}$ long and $6 \mathrm{~cm}$ in diameter clad with thin tantalum and with a tantalum flange $1.5 \mathrm{~cm}$ thick and $11 \mathrm{~cm}$ in diameter at the back of each target. Each target was enclosed in a stainless-steel 'sleeve' $2.6 \mathrm{~cm}$ thick, but the tantalum flange (at the back) remained outside the shield. The cylindrical transportation flask (axis vertical) had lead walls $16.8 \mathrm{~cm}$ thick with inner and outer steel skins $0.6 \mathrm{~cm}$ and $1.6 \mathrm{~cm}$ thick. In addition, all three targets were surrounded by an aluminium 'liner' $3 \mathrm{~cm}$ thick. A schematic representation is given in Figure 2.

The data presented in Table 1 were initially analysed following the method described in [2] whereby the relative strengths of gamma-ray lines from a one-radionuclide source were used to determine the thickness of an absorber between the source and the detector through the variation with energy of the gamma-ray mass attenuation coefficient $\mu / \rho(k)$ where $k$ is the gamma-ray energy. Following the method, $c(k)=$ $\varepsilon(k) \exp (-\mu / \rho(k) \rho t) \alpha(k) a$ was fitted at energies $k_{j}$ to the observed count rates $C_{j} \quad$ with thickness $\rho t$ and activity $a$ as parameters ( $\varepsilon=$ detector efficiency, $\alpha=$ gamma-ray emission probability). The absolute efficiency of the HPGe detector was evaluated according to systematics [5] for a source-to-detector distance of $99 \mathrm{~cm}$ since the flask outer diameter is $98.4 \mathrm{~cm}$ and the HPGe was $50 \mathrm{~cm}$ from the flask, gamma-ray mass attenuation coefficients were taken from NIST's tables [6] of X-ray attenuation coefficients, and emission probabilities were taken from ENDF/B-VII decay files [7].

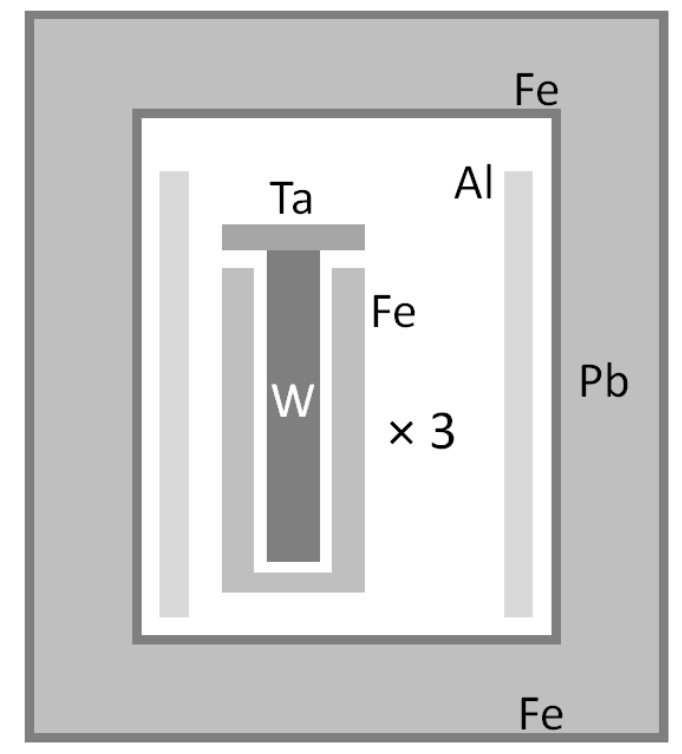

Figure 2. Schematic representation of three TS-2 targets inside transport flask - not to scale. Dimensions and materials are given in the text.

Applied to the twenty ${ }^{172} \mathrm{Lu}$ lines in Table 1 this initial analysis resulted in an absorption thickness (in terms of lead) of $20.1 \pm 0.3 \mathrm{~cm}$ and an activity of $73 \pm 3 \mathrm{GBq}$. It can now be asked how well this activity derived from gamma-ray spectroscopy compares with the activity that was declared when the transportation flask was loaded with the three targets. 
Since it was impractical to make detailed activity measurements on the bare targets, the declared radionuclide inventories of the three targets in the transportation flask relied on calculations carried out [8] using the MCNPX Monte Carlo code ${ }^{2}$ [9] in association with the CINDER'90 transmutation code [10]. The geometry and materials for the calculations were taken from a detailed model of the TS-2 targetreflector-and-moderator (TRAM) system built up using CombLayer [11], a set of C++ programs in which the model geometry is effectively written into the $\mathrm{C}++$ construction system and which is then compiled and executed to produce the MCNPX input file. This object-oriented approach allows rapid production of complex MCNPX models where the majority of engineering details can be described in detail. In addition, for the calculations described herein, a sub-set of $\mathrm{C}++$ programs in the CombLayer was used to prepare the neutron tallies and other data needed for subsequent runs (using a set of 'in-house' bash-shell scripts) of the transmutation code. The result of the Monte Carlo calculations was a total of $425 \mathrm{GBq}$ of ${ }^{172} \mathrm{Lu}$ in the three targets [12] - quite different from the measured activity of $73 \pm 3 \mathrm{GBq}$.

At first sight, therefore, there seems to be a significant discrepancy between the calculated and measured activities. However, not all the information known about the composite radioactive item in the flask has yet been used. Specifically, from Figure 2, and from the declaration that would accompany the shipment, it is known that there are three targets inside the flask, and it is evident that these targets can be considered as two distinct composite objects - the first object being the set of three target bodies inside their thick sleeves, and the second object being the set of three flanges outside the sleeves. Accordingly, the set of observed counts rates $C_{j}$ in Table 1 was then fitted by $c(k)=\varepsilon(k) \alpha(k)\left(\exp \left(-\mu / \rho(k) \rho t_{1}\right) a_{1}+\exp \left(-\mu / \rho(k) \rho t_{2}\right) a_{2}\right) \quad$ in order to extract two source strengths $a_{1}$ and $a_{2}$ and two absorption thicknesses $\rho t_{1}$ and $\rho t_{2}$. The results are shown in Table 2 , and in Table 3 the thicknesses and activities extracted from the fits are compared with the actual thicknesses and the activities from Monte Carlo calculations.

\begin{tabular}{|c|c|c|c|c|c|c|c|c|c|c|c|}
\hline \multicolumn{4}{|c|}{ First source and absorber } & \multicolumn{5}{c|}{ Second source and absorber } \\
\hline \multicolumn{3}{|c|}{ Lead thickness $(\mathrm{cm})$} & \multicolumn{3}{|c|}{ Activity $(\mathrm{GBq})$} & \multicolumn{2}{c|}{ Lead thickness $(\mathrm{cm})$} & \multicolumn{3}{|c|}{ Activity $(\mathrm{GBq})$} \\
\hline & \pm int & \pm ext & & \pm int & \pm ext & & \pm int & \pm ext & & \pm int & \pm ext \\
\hline 23.4 & 0.2 & 1.2 & 328 & 45 & 213 & 16.0 & 0.6 & 3.0 & 2 & 1 & 6 \\
\hline 23.3 & \multicolumn{2}{|c|}{0.3} & 368 & \multicolumn{2}{|c|}{56} & 14.5 & \multicolumn{2}{c|}{0.5} & 0.7 & \multicolumn{2}{c|}{0.3} \\
\hline
\end{tabular}

Table 2. Results of fitting observed ${ }^{172} \mathrm{Lu}$ line strengths to extract two source strengths and two absorber thicknesses. The upper line of results was obtained using the minimisation routine VA04A [13], and the uncertainties in the fitted parameters were obtained by repeatedly perturbing all the data points by normally distributed random numbers matched to the uncertainties in the data points and refitting, and then taking the standard deviations of the resultant sets of 'perturbed' fitted parameters ('ext' (external consistency) values of uncertainty $\sqrt{\chi_{\text {pdf }}^{2}} \times$ 'int' (internal consistency) values of uncertainty). The lower line of results was obtained using the CERN minimisation tool MINUIT [14].

\footnotetext{
${ }^{2}$ MCNPX version 2.7.0 was used, along with the CEM03 physics model, and MCNPX was forced to use tables (if available) for neutron interactions up to the CINDER'90 upper limit of $25 \mathrm{MeV}$.
} 


\begin{tabular}{|c|c|c|c|c|c|c|c|}
\hline \multicolumn{2}{|c|}{ Target bodies $(\mathrm{GBq})$} & \multicolumn{2}{|c|}{ Thickness $(\mathrm{cm})$} & \multicolumn{2}{|c|}{ Target flanges $(\mathrm{GBq})$} & \multicolumn{2}{|c|}{ Thickness $(\mathrm{cm})$} \\
\hline $\begin{array}{c}\text { Monte } \\
\text { Carlo }\end{array}$ & $\begin{array}{c}\text { From } \gamma \\
\text { spec. }\end{array}$ & Actual & $\begin{array}{c}\text { From } \gamma \\
\text { spec. }\end{array}$ & $\begin{array}{c}\text { Monte } \\
\text { Carlo }\end{array}$ & $\begin{array}{c}\text { From } \gamma \\
\text { spec. }\end{array}$ & Actual & $\begin{array}{c}\text { From } \gamma \\
\text { spec. }\end{array}$ \\
\hline $425 \pm 85$ & $350 \pm 80$ & $20.7 \pm 0.2$ & $23.5 \pm 1.0$ & $0.4 \pm 0.2$ & $1.2 \pm 1.0$ & $19.0 \pm 0.2$ & $15 \pm 2$ \\
\hline
\end{tabular}

Table 3. Comparison of actual absorber thicknesses and Monte Carlo calculations of the sums of ${ }^{172} \mathrm{Lu}$ activities in three target bodies and in three target flanges with thicknesses and activities extracted from fits to ${ }^{172} \mathrm{Lu}$ gamma-ray lines measured outside the flask (the uncertainties given for the actual thicknesses were estimated from manufacturers' drawings). The uncertainty given for each of the measured quantities is a single-number compromise amongst the corresponding uncertainties in Table 2, and should be interpreted as such. The uncertainties given for the Monte Carlo results are plausible estimates of the uncertainties in the modelling and the nuclear data.

The ${ }^{172} \mathrm{Lu}$ activity in the target bodies extracted from the fits agrees well with the Monte Carlo calculation of ${ }^{172} \mathrm{Lu}$ activity, and the thickness of the first absorber extracted from the fits compares well with the 'lead equivalent' of the total thickness of absorber between the target bodies and the detector as shown in Table 4, especially when it is remembered that on average the distance travelled by gamma-rays from the targets to the detector is necessarily a little greater than the radial thickness of the absorbers. The thickness of the second absorber extracted from the fits is less than the total thickness of absorber between the target flanges and the detector, but the uncertainty on this thickness is greater than it is for the first absorber, and the activity of this second source of ${ }^{172} \mathrm{Lu}$ activity is much less than the activity of the first source. It would appear, therefore, that fitting two absorber thicknesses and two activities to the spectrum of gamma-rays transmitted through the thick walls of the flask gives results that agree surprisingly well with the known dimensions of the flask and the activities inside the flask.

\begin{tabular}{|lcc|}
\hline Absorber & Thickness $(\mathrm{cm})$ & Lead equiv. $(\mathrm{cm})$ \\
Lead wall & $16.8 \pm 0.1$ & 16.8 \\
Steel skins & $2.2 \pm 0.1$ & 1.5 \\
Aluminium liner & $3.0 \pm 0.1$ & 0.7 \\
Steel sleeve & $2.6 \pm 0.1$ & 1.7 \\
& & Total $20.7 \pm 0.2$ \\
\hline
\end{tabular}

Table 4. Comparison of total absorber thickness between target bodies and detector, where 'lead equivalent' thicknesses have been derived simply by scaling by densities $(2.7,7.5$ and $11.3 \mathrm{~g} \mathrm{~cm}^{-3}$ for $\mathrm{Al}$, steel and $\mathrm{Pb}$ respectively).

Of course, approximations are being made: for example, the 'body' and 'flange' activities have simply been lumped together, absorptions have been parametrised as single exponential factors, and the same point-source-to-detector efficiency function has been used for the lumped bodies and flanges. If the fitting process were being done 'properly', calculations in much more detail would have had to be done beforehand. But if measurements by gamma-ray spectroscopy are to be made to support the declared identity of a highly radioactive item or set of items behind a thick shield, the data must be analysed reasonably quickly to yield a useful result; long delays while a 'proper' analysis was carried out would be unlikely to be tolerable. 
Consequently, in practice, approximations such as are being made here would probably inevitably be made, but nevertheless in the present case the use of approximations based on prior information has succeeded in achieving good agreement between measured activities and declared activities.

It may be asked whether the work described in this publication could be developed further or applied elsewhere, or whether the work became possible simply because of a fortuitous set of circumstances that are unlikely to occur again. Clearly it was fortunate that many gamma-ray lines from one radionuclide spanning a wide energy range were observable, and also that it was already known that there were essentially two separate lumped sources of gamma-rays, each one behind a different absorber. And, of course, the gamma-ray spectrum had to be accumulated over a relatively long time.

Nevertheless, the present publication shows that it may be possible to exploit highresolution gamma-ray spectroscopy of highly radioactive items behind thick shielding to a greater extent than might appear possible at first sight. It has been shown that the relative strengths of gamma-ray lines measured through thick shielding material can be used to estimate the thickness of the shielding material and therefore to estimate the source strength behind the shielding material by correcting for the attenuation in the shielding material, and that such estimates can be refined if something is known of the geometry of the radioactive material. Such techniques could find application for quality-checking purposes such as:

○ when ${ }^{60} \mathrm{Co}$ for sterilisation plants, spent fuel rods from nuclear power stations, or highly active products from reprocessing plants are being moved by road or rail, and it is desirable to make whatever checks on the contents are possible without opening up the flasks; or

o when it is desirable to check that two physically different assemblies of highly radioactive material have not been loaded into the wrong flasks; or

○ when illicit trafficking of nuclear and radiological materials is suspected.

\section{Summary and conclusion}

In this publication it is not the intention to claim that a reliable new method has been found for measuring activities quantitatively behind thick shields. But it may not be unreasonable to suggest that measurements and analyses such as are presented herein could, in some circumstances, provide evidence in favour of or against estimates of activity obtained in other ways.

\section{References}

[1] 'Detecting nuclear and radiological materials', Royal Society policy document 07/08, March 2008.

[2] D J S Findlay, G P Škoro, G J Burns and S Ansell, Appl. Radiat. Isot. 125 (2017) 1.

[3] http://www.isis.stfc.ac.uk/.

[4] http://www.gammadata.se/assets/Uploads/Falcon-SS-C38597.pdf.

[5] D J S Findlay, Radiat. Meas. 94 (2016) 23. 
[6] https://www.nist.gov/pml/x-ray-mass-attenuation-coefficients.

[7] http://www.nndc.bnl.gov/endf/b7.1/download.html.

[8] G P Škoro, 'Storage/transport flask for ISIS TS-2 targets', ISIS internal report, 2014.

[9] MCNPX 2.7.0 - Monte Carlo N-Particle Transport Code System for Multiparticle and High Energy Applications, https://mcnpx.lanl.gov/.

[10] W L Wilson et al., Proc. SARE4 Workshop, Knoxville, USA, 1998.

[11] S Ansell, Proc. ICANS XXI Meeting, Mito, Japan, 2014, JAEA-Conf 2015002, pp. 148-154. Also https://github.com/SAnsell/CombLayer.

[12] D J S Findlay, 'TS-2 W\#1-W\#3 target activities and decay heats', ISIS internal report ISIS-DJSF-17-07-A, 2017.

[13] Harwell Subroutine Library, http://www.hsl.rl.ac.uk/.

[14] http://hep.fi.infn.it/minuit.pdf. Also http://cernlib.web.cern.ch/cernlib/. 


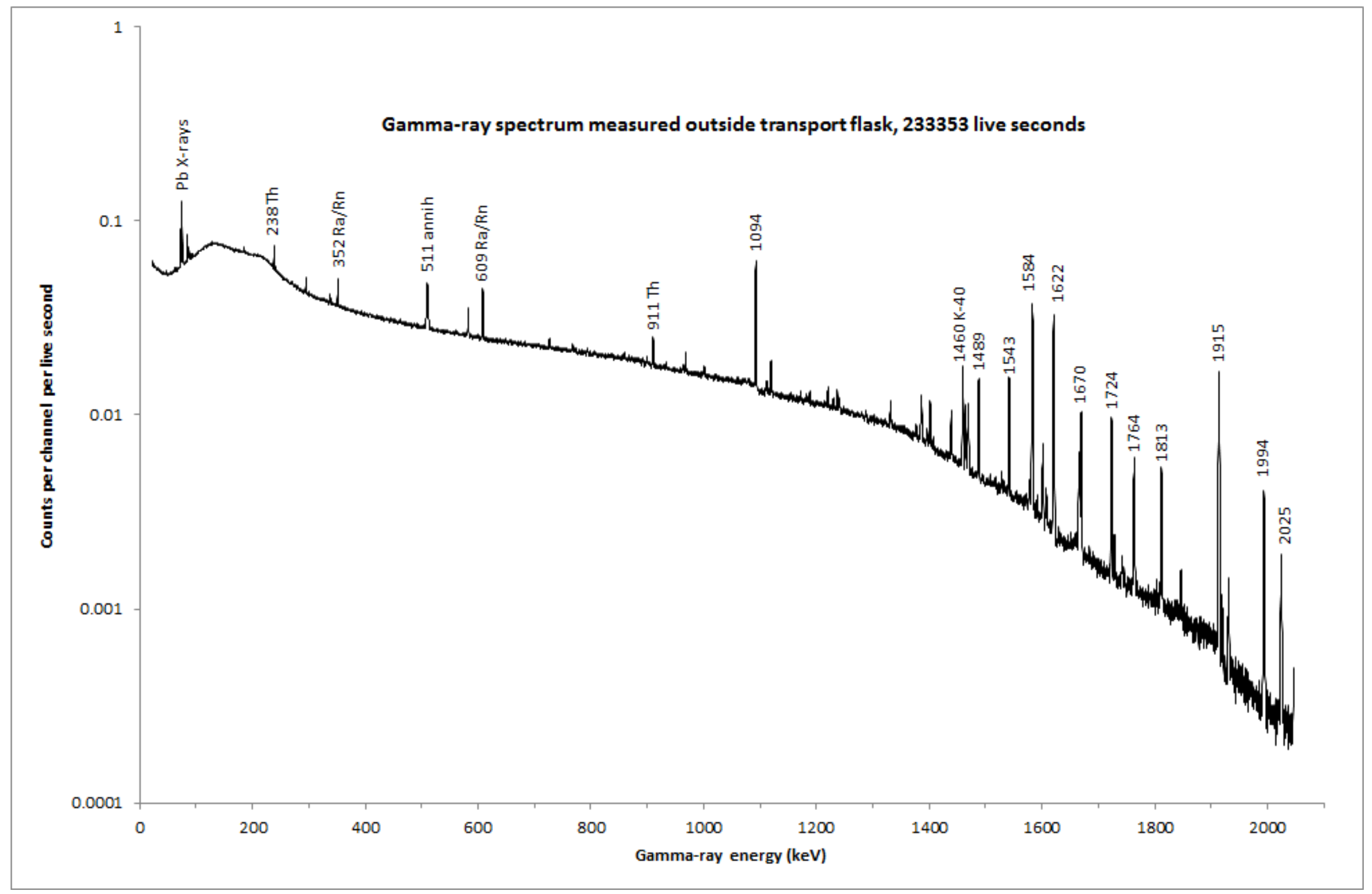

Figure 1. Spectrum of gamma-rays measured outside the transportation flask containing the three spallation targets. The strongest lines are labelled with their energy in $\mathrm{keV}$. Lines with energy alone, ${ }^{172} \mathrm{Lu}$; 'Th' denotes ${ }^{232} \mathrm{Th}$ daughters; ${ }^{2226} \mathrm{Ra} /{ }^{222} \mathrm{Rn}$ ' denotes radium and radon daughters; in the energy range below the 1094-keV line all visible lines are from external natural background. 